\title{
Interactive comment on "An
}

\section{observationally-constrained estimate of global dust aerosol optical depth" by David A. Ridley et} al.

\section{Anonymous Referee \#3}

Received and published: 16 June 2016

In this manuscript the authors present a global reconstruction of dust AOD based on satellite data and sun photometer retrievals, using sun photometer data to correct satellite bias and various model simulations to separate the regional contribution of dust from other aerosols. This is a really nice manuscript with some good ideas and a dataset the has the potential to be a widely cited reference. Because of this potential it is necessary to be extra careful, though. The authors have followed previous methodology, including the weaknesses. l'd like to see these addressed before I support the publication of this manuscript.

Major Comments: 1. One of my major concerns is the use of the different emission schemes in different models. This will have an impact on the calculation of the dust 
AOD (eq. 1). How much of the model-ensemble uncertainty is due to different emission schemes and how much due to inter-model variability?

2. I haven't found an explanation why the AOD reconstruction is limited to the 15 regions. Why do you not reconstruct AOD over the whole globe and show it on a map (e.g. using a yearly median)? You can still only calculate the correction factor using the dust-dominated regions.

Interactive

comment

3. The assumption on which the correction factor are based seem weak to me. It is true that most of the global dust AOD is dominated by the North African and East Asian region. But this doesn't mean that you only need to concentrate on a few region, but that the spatial distribution is no Gaussian. In fact, if you look at a histogram of a snapshot in time you will probably find that dust is spatially log-normally distributed. My suggestion to the authors is to look at the spatial distribution of the satellite and sun photometer data and if it's lognormal, try to take the logarithm of all initial AOD data such that it is spatially normally distributed and rethink their calculations (especially equations 1-3 and Figure 2) and discussion from that perspective.

4. Global means make sense for GHG but not for aerosols. Talking about a global mean $A O D$ is meaningless. It gives you absolutely no information about what the AOD could be on any point on Earth. I know everybody's doing it and there's a weak argument that can be made for inter-paper comparison's sake. But this manuscript has the potential to be a widely cited reference and it has the means to provide data for more regionally-based comparisons in the future. Figure 4 looks very fancy but gives very little useful information. Maybe in addition to Figure 4 that compares with previous papers you could prepare a synthesis figure or table with which people writing papers in the future can easily compare their results (something like figure 9 but less messy no offense to figure 9 ).

Printer-friendly version

Minor comments:

Discussion paper

The references to air quality and health seem out of place in this manuscript. There is 
no need to mention these aspect if they are not discussed anywhere.

Page 2, lines 7-9: I don't know if that's a mistake in the original Huneeus paper, but if ACPD you give the median because the distribution is not Gaussian, then you shouldn't give the standard deviation, which is a parameter in the Gaussian distribution. Chapters 2.1, 2.2, 2.3: I would appreciate it if the description of errors was consistent between the three instruments. Page 6\&7, lines 32-7: Looking at the data in Figure 2 I would guess that the data is not normally distributed. The choice of a linear regression to calculate the bias between AERONET and satellites is therefore doubtful. See my major comment 3. Page 8, Eq.1: In my experience, aerosol concentrations, loads, and therefore AOD are not normally distributed in space. The mean AODs calculated here may not be representative of the central tendency in each region. See major comment 3. Page 10 line 3: $A E<0.4$ Figure 2: In the MISR panel, there are values only for one of the two regressions. Also I can see only one regression line

Interactive comment on Atmos. Chem. Phys. Discuss., doi:10.5194/acp-2016-385, 2016. 